\title{
We should be considering age, comorbidities and health-care system performance to relax Covid-19 related social distancing measures
}

\author{
Bruno de Paula ${ }^{1}$, Renan Carraretto ${ }^{2}$, and Nathalia Barreto ${ }^{3}$ \\ ${ }^{1}$ Cambridge University Hospitals NHS Foundation Trust \\ ${ }^{2}$ Universidad Privada del Este \\ ${ }^{3}$ Hospital Pro-Cardiaco
}

May 14, 2020

We should be considering age, comorbidities and health-care system performance to relax Covid-19 related social distancing measures

Dear Editor,

Aiming to avoid a health system collapses due to the COVID-19 pandemic; social distancing measures are taken broadly. ${ }^{1}$ The very high or high-risk population is of specific concern, once expected to have an increase rate of admission. ${ }^{2}$ Besides criticised, the strategy seems to be working properly and now brings the discussion about when the measures will be relaxed with safety.

Comparisons with other pandemics are not entirely appropriate, but it seems reasonable to look back at the past and consider valuable lessons learned. The direct association between death and age as well as for comorbidities or clinical risk is observed in other viral infections similarly to the current outbreak. ${ }^{3-13}$ Age is also a known as an independent survival factor. On the other hand, it should be highlighted that young children are the exception once have an immature immune system, main reasons why we generally see a "J-like" curve shape as schematically represented by the Figure 1. Although, age-mortality rate seems to be similar amongst world regions, the poor healthcare and quality index is associate with increase death rate. ${ }^{14}$

Unfortunately, we were relying on certainties that are fragile for the moment. There is no solid evidence about herd immunity in patients with asymptomatic disease. Moreover, we all expect that the patients that recovered from the infection are hopefully immune or at least have a mild disease in case of re-infection but the knowledge about the immune response and anti-bodies nature is currently imature ${ }^{15,16}$. There is also scare evidence about mass use disposable barrier methods, such as face masks or gloves, will diminish the viral transmission. ${ }^{17}$ Although community use of personal protection equipment might give a sensation of safety in some cultures, the misuse or re-use could be dangerous and there is no evidence assuring the benefit provided by community manufactured masks. ${ }^{18,19}$

Therefore, once the evidence is being gathered and a vaccine, probably the most reliable scientific based way out of this crisis, will take at least some months to be available, we should consider relax social distancing measures based on age adjusted by comorbidities as a first step whilst continuing with hand washing associated with high standards of self-hygiene when possible.

Bruno H R de Paula ${ }^{*}$, Renan G A Carraretto ${ }^{2}$, Nathalia M P B Barreto 3

1 Department of Oncology, Addenbrooke's Hospital, Cambridge University Hospitals NHS Foundation Trust,Cambridge CB2 0QQ, UK

2 Universidad Privada del Este, Ciudad Presidente Franco - Paraguay 
${ }^{3}$ Division of Cardiology, Hospital Pró-Cardiaco, Rio de Janeiro, Brazil

* Correspondent author

Competing Interests

The authors declare that they have no competing interests

References:

1. Lewnard, Joseph A., and Nathan C. Lo. "Scientific and ethical basis for social-distancing interventions against COVID-19." The Lancet. Infectious diseases (2020).

2. nhs.uk. 2020. Who's At Higher Risk From Coronavirus - Coronavirus (COVID-19) . [online] Available at: <https://www.nhs.uk/conditions/coronavirus-covid-19/people-at-higher-risk-from-coronavirus/whos-athigherrisk-from-coronavirus/ $>$ [Accessed 4 May 2020].

3. Ramiro, Diego, et al. "Age-specific excess mortality patterns and transmissibility during the 1889-1890 influenza pandemic in Madrid, Spain." Annals of epidemiology 28.5 (2018): 267-272.

4. Cilek, Laura, Gerardo Chowell, and Diego Ramiro Fariñas. "Age-specific excess mortality patterns during the 1918-1920 influenza pandemic in Madrid, Spain." American journal of epidemiology187.12 (2018): 25112523.

5. Viboud, Cécile, et al. "Global mortality impact of the 1957-1959 influenza pandemic." The Journal of infectious diseases 213.5 (2016): 738-745.

6. Ma, Junling, Jonathan Dushoff, and David JD Earn. "Age-specific mortality risk from pandemic influenza." Journal of theoretical biology 288 (2011): 29-34.

7. Donaldson, Liam J., et al. "Mortality from pandemic A/H1N1 2009 influenza in England: public health surveillance study." Bmj 339 (2009): b5213.

8. Chan-Yeung, Moira, and Rui-Heng Xu. "SARS: epidemiology." Respirology 8 (2003): S9-S14.

9. Verity, Robert, et al. "Estimates of the severity of coronavirus disease 2019: a model-based analysis." The Lancet Infectious Diseases (2020).

10. Glynn, Judith R. "Protecting workers aged 60-69 years from COVID-19." The Lancet Infectious Diseases (2020).

11. Onder, Graziano, Giovanni Rezza, and Silvio Brusaferro. "Case-fatality rate and characteristics of patients dying in relation to COVID-19 in Italy." Jama (2020).

12. www1.nyc.gov. 2020. COVID-19: Data - NYC Health. [online] Available at:

$<$ https://www1.nyc.gov/site/doh/covid/covid-19-data.page> [Accessed 4 May 2020].

13. Cromer, Deborah, et al. "The burden of influenza in England by age and clinical risk group: a statistical analysis to inform vaccine policy." Journal of Infection 68.4 (2014): 363-371.

14. Global - Paget, John, et al. "Global mortality associated with seasonal influenza epidemics: New burden estimates and predictors from the GLaMOR Project." Journal of global health 9.2 (2019).

15. Wu, Fan, et al. "Neutralizing antibody responses to SARS-CoV-2 in a COVID-19 recovered patient cohort and their implications." (2020).

16. Altmann, Daniel M., Daniel C. Douek, and Rosemary J. Boyton. "What policy makers need to know about COVID-19 protective immunity." The Lancet (2020).

17. Cheng, Kar Keung, Tai Hing Lam, and Chi Chiu Leung. "Wearing face masks in the community during the COVID-19 pandemic: altruism and solidarity." The Lancet (2020). 
18. Leung, Chi Chiu, Tai Hing Lam, and Kar Keung Cheng. "Mass masking in the COVID-19 epidemic: people need guidance." Lancet 395.10228 (2020): 945.

19. Liu, Xiaopeng; Zhang, Sisen. COVID-19: face masks and human-to-human transmission. Influenza And Other Respiratory Viruses,[s.l.], p. 1-2, 5 abr. 2020. Wiley. http://dx.doi.org/10.1111/irv.12740.

\section{Hosted file}

Figure 1 a,b and c.docx available at https://authorea.com/users/321987/articles/451108-weshould-be-considering-age-comorbidities-and-health-care-system-performance-to-relaxcovid-19-related-social-distancing-measures 